\title{
When do Word Embeddings Accurately Reflect Surveys on our Beliefs About People?
}

\author{
Kenneth Joseph \\ Computer Science and Engineering \\ University at Buffalo \\ Buffalo, NY, 14226 \\ kjoseph@buffalo.edu
}

\author{
Jonathan H. Morgan \\ University of Applied Sciences \\ Fachhochschule Potsdam \\ Potsdam, Germany \\ morgandfh-potsdam. de
}

\begin{abstract}
Social biases are encoded in word embeddings. This presents a unique opportunity to study society historically and at scale, and a unique danger when embeddings are used in downstream applications. Here, we investigate the extent to which publicly-available word embeddings accurately reflect beliefs about certain kinds of people as measured via traditional survey methods. We find that biases found in word embeddings do, on average, closely mirror survey data across seventeen dimensions of social meaning. However, we also find that biases in embeddings are much more reflective of survey data for some dimensions of meaning (e.g. gender) than others (e.g. race), and that we can be highly confident that embedding-based measures reflect survey data only for the most salient biases.
\end{abstract}

\section{Introduction}

In April of 2015, protests erupted over the murder of Freddie Gray, Jr. Questions about what to call those protesting quickly became the focus of a national debate. In an interview on $C N N$ with Erin Burnett, ${ }^{1}$ Baltimore City Councilman Carl Stokes admonished then-President Barack Obama and then-Mayor Stephanie Rawlings-Blake for using the word thugs to refer to the protesters. Burnett challenged Stokes' admonition, claiming the protesters were indeed thugs because "They know it's wrong to steal and burn." Stokes responded by stating the protesters were "...children who have been set aside [and] marginalized."

The argument between Burnett and Stokes is over the way we label people, the meanings of those labels, and the impacts these meanings can have. Councilman Stokes wants to avoid using the label "thug" because of its established, negative

\footnotetext{
${ }^{1}$ http://nymag.com/intelligencer/2015/04/carl-stokes-tocnn-thug-is-racially-charged.html
}

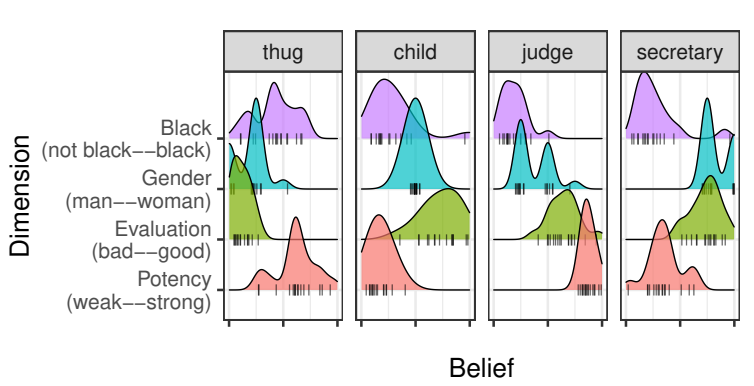

Figure 1: Beliefs (x-axis) about four identities (separate plots) along four dimensions of social meaning ( $y$ axis). Beliefs are displayed as distributions of survey responses. The scale is different for each dimension, e.g. for Evaluation, survey participants responded with their belief as to whether people who held the identity were more likely to be a bad (further left on the x-axis) or good (further right).

connotation towards black Americans (Dow, 2016). The survey data collected for this paper, a sample of which is shown in Figure 1, provides further evidence of this association between thugs and black Americans. Respondents to our survey, on average, expected thugs to be bad, and that approximately $42.4 \%$ of thugs would be black. Of the 57 identities we studied, the only identity perceived to be more black was criminal, at $47.3 \%$.

The beliefs we have about people who hold particular identities (McCall and Simmons, 1978) are important, because they often determine the behaviors we take towards people who are labeled with those identities (Ridgeway and Smith-Lovin, 1999). ${ }^{2}$ For example, as Councilman Stokes knows,

\footnotetext{
${ }^{2}$ Different kinds of beliefs about identities have different names. For example, contextualized beliefs are called impressions (Heise, 1987), and aggregations of beliefs across multiple dimensions of meaning are called stereotypes (Fiske et al., 2002). The beliefs we study here are typically called sentiments or associations. However, given the distinct meaning of these terms in NLP, we use the general term "belief" in this paper. This aligns roughly with the generic use of the terms "bias" and "stereotype" in related NLP literature.
} 
we do not behave the same way towards children as we do towards thugs. This is because, as reflected in Figure 1, people generally believe that children are weak and good, whereas thugs are bad and powerful. This leads us to want to do things like help children, versus wanting to attack thugs (Heise, 2007).

However, measuring beliefs is difficult. Traditionally, we have relied on surveys to collect these measurements. But there are tens of thousands of identities (Joseph et al., 2016; MacKinnon and Heise, 2010), and beliefs about them can form along many different dimensions of sociocultural meaning (e.g. gender, race, and others displayed in Figure 1). Measuring beliefs about many identities, on many dimensions, using traditional surveys can therefore be difficult. Further, measuring the evolution of beliefs is often impossible with surveys, because survey data is extremely sparse historically (Garg et al., 2018). Finally, measuring how these beliefs change with additional contextual information (e.g. beliefs about specific teachers, rather than teachers in general) is notoriously difficult with survey data (Heise, 2007).

Recognizing these difficulties, scholars have begun to develop NLP tools to measure beliefs about identities historically, at scale, and in context (Joseph et al., 2017; Hoyle et al., 2019; Fast et al., 2016; Garg et al., 2018; Field et al., 2019). Most recent methods derive these measures by manipulating word embeddings. Studying beliefs enmeshed in word embeddings is also critical because embeddings are widely used in downstream NLP models, which are themselves beginning to label people, for example, as job-worthy or not (De-Arteaga et al., 2019). Measuring beliefs about people using embeddings therefore serves the dual purpose of understanding human biases and of ensuring such biases are not propelled further along by algorithms.

However, work remains to understand when embedding-based measures of beliefs about identities accurately reflect more traditional survey measures, and why some beliefs may be reflected more accurately than others. The present work combines new and existing survey data with an extensive set of embedding-based measurement strategies to explore this at both the dimension level and the belief level. At the dimension level, for example, we ask, how well do embeddings capture beliefs about gender, relative to race? And if differences exist, why? Such issues have arisen in existing work, for example, where Garg et al. (2018) see correlations of .65 between embedding-based and survey-based measures of beliefs about gender, but only .15 for ethnicity-based beliefs. At the beliefs-level, we ask, for example, how much more accurately do we capture beliefs about the Potency (strength) of thugs, relative to beliefs about the Potency of children? Accuracy at this level is critical for linking historical trends in social behavior to societal-level beliefs about particular identities.

Our primary contributions are as follows:

- We show that what we measure is more important than how we measure it in determining the correlation between embedding-based and survey-based measures of beliefs about people.

- At the dimension level, the beliefs we measure most accurately are also the most important for how we label others.

- At the belief level, assuming we can identify a good measurement model, embedding-based measures are significantly more accurate for more extreme, and more agreed upon, beliefs. All code and data necessary to replicate the analyses in this article can be found at https://github. com/kennyjoseph/embedding_impressions.

\section{Related Work}

Our work is grounded in literature on measuring beliefs about identities in social psychology in general and, more specifically, via word embeddings. We address these two literatures separately here.

\subsection{Belief Measurement in Social Psychology}

A common approach for measuring beliefs about specific identities is to assume a dimensional representation - that is, to assume a set of distinct dimensions of social meaning can be used to characterize how we think and feel about someone that holds a particular identity. From this dimensional perspective, two primary questions arise.

First, what are the dimensions along which beliefs form? Social psychologists have identified three classes of important dimensions: traits, affective meanings, and semantic associations. Traits represent visible-although also socioculturally defined — characteristics like age, gender, and race (Freeman and Ambady, 2011). Affective dimensions of social meaning represent how we feel about a given person and/or identity (Todorov et al., 2015; Fiske et al., 2002; Heise, 2007). Here, 
we use the three affective dimensions proposed by Heise (2007) and that are popular in sociology (Rogers et al., 2013) - Evaluation (goodness/badness), Potency (strength/weakness), and Activity (active/passive). Finally, social psychologists often characterize beliefs about identities in terms of semantic associations to particular concepts (Freeman and Ambady, 2011) or institutions (MacKinnon and Heise, 2010). For example, people link the identities brother and sister together because they are both associated with the family institution. In the present work, we collect beliefs for seventeen different dimensions of social meaning, incorporating age, race, gender, evaluation, potency, activity, and six institutional associations.

Second, given a theorized dimension of meaning, how should we measure society-wide beliefs about where particular identities lie on that dimension? Here, we adopt perhaps the most common approach, which uses semantic differential scales on surveys (Osgood et al., 1975). The semantic differential technique asks respondents to place an identity on a sliding scale with two opposing concepts (e.g. weak and strong, see the example in Figure 2A). Finally, it is worth noting that here, like in most social psychology research, we assume that responses from survey participants generalize to American culture writ large. This assumption is built on the well-established culture-as-consensus paradigm in psychological anthropology (Karabatsos and Batchelder, 2003; Batchelder and Romney, 1988), and empirical work showing that people tend to agree on the vast majority of their beliefs about people (Heise, 2007). Nonetheless, many counterexamples exist (Berger et al., 1992; Smith-Lovin and Douglas, 1992). We leave questions about how to address these issues to future work.

\subsection{Measuring beliefs with embeddings}

Embedding-based approaches to measuring beliefs typically follow a three step process of corpus/embedding selection, dimension selection, and word position measurement.

Corpus/Embedding Selection Several recent works have argued that the corpus used can impact measures of beliefs about people derived from word embeddings (Lauscher and Glavaš, 2019; Mirzaev et al., 2019; Sweeney and Najafian, 2019). For example, Brunet et al. (2019) show how to reduce gender bias in embeddings by removing particular documents from a corpus. However, several oth- ers have shown that in their analyses, the corpus used does not significantly impact results (Spirling and Rodriguez, 2019; Garg et al., 2018; Kozlowski et al., 2019; Caliskan et al., 2017). Differences in the embedding model used have also been observed to impact measurements (Chaloner and Maldonado, 2019). Again, though, robustness checks from other studies suggest a limited effect beyond the somewhat general hyperparameters of window size and the number of dimensions estimated (Garg et al., 2018; Kozlowski et al., 2019).

Dimension Selection To measure beliefs, one first must select a dimension along which the belief is assumed to be held. Much of the literature has focused on dimensions related to gender or race. Others, however, have seen value in moving beyond these dimensions (Agarwal et al., 2019; Sweeney and Najafian, 2019). Most relevant is the work of Kozlowski et al. (2019), who study the association of 59 concepts across 20 different dimensions of sociocultural meaning, and that of An et al. (2018), who induce 732 different dimensions using WordNet to study contextual effects of linguistic meaning. While neither work focuses heavily on identities, these efforts compliment our goal of studying a broad range of dimensions of social meaning.

Scholars then identify a direction within the embedding that represents this dimension. To do so, an approach similar to the semantic differential idea is used. Terms are selected to represent the two ends of the dimension. For example, to identify the gender direction, words at one end might be he and him, and words at the other end, she and her. Scholarship varies on how these dimensioninducing word sets are selected. For example, several scholars have used demographically gendered and/or racialized names (Bolukbasi et al., 2016; Caliskan et al., 2017), while others have relied on careful extraction of concepts from dictionaries and thesauri (Kozlowski et al., 2019). Kozlowski et al. (2019) find that having more words at each end generally provides better measurements, and others have found a need to use frequently occurring terms (Ethayarajh et al., 2019; Brunet et al., 2019). Beyond these observations, however, scholars have generally found stable results as long as reasonable word sets are selected.

Word Position Measurement Finally, the position of each identity along this direction must be identified. Doing so entails two major deci- 
sions. First, how should one quantify the direction, given the dimension-inducing words? For example, Bolukbasi et al. (2016) identify the direction by taking the first dimension of a PCA on the full set of direction words. Second, how should one define the position of points along this line? For example, several works use the cosine similarity between the identified "bias direction" and the embedding of each identity. Scholars have also recently proposed supervised methods for word position measurement (Sweeney and Najafian, 2019; Agarwal et al., 2019). Such approaches are important, but assume the existence of some training data, which may or may not be available in certain measurement contexts We therefore do not explore these methods further in the present work.

In sum, using embeddings to measure beliefs requires a series of decisions, the impacts of which are still debated. Below, we provide the most comprehensive study to date on the importance of these decisions on measurement quality.

\section{Survey Data}

We collect two new survey datasets for this paper. The first measures beliefs about the 57 identities $^{3}$ in Table 1 on seventeen dimensions of social meaning described below. The second measures the ways in which a set of survey respondents label people with these identities in hypothetical social situations.

We used a cluster-based approach to select the 57 identities we study. We study nine sets of six identities, where each set has been shown in prior work to be related in some way. Five of the sets are characterized by a salient association to a specific institution described by MacKinnon and Heise (2010). Three sets are characterized by salient trait similarities and differences on gender, age or race/ethnicity. And one set of identities is included where all identities have strong negative Evaluations. Finally, we include three random identities as a mechanism for comparison in other work not described here. For further details on the selection criteria, survey populations, and results, see the Appendix.

\subsection{New Belief Measurement Data}

We collected survey data on beliefs about identities from 247 respondents on Amazon's Mechanical Turk. Each survey respondent provided re-

\footnotetext{
${ }^{3}$ Because not all embedding models account for bigrams, we removed "police officer" from all analyses in this paper. However, for future purposes, we include it in our description of the data here.
}

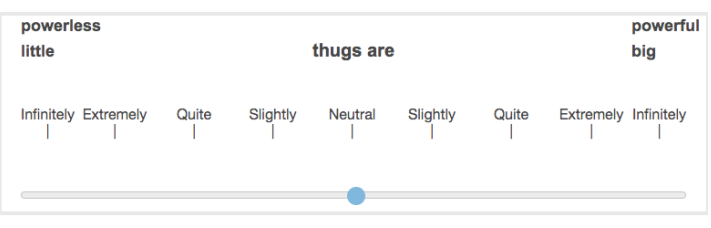

(a)

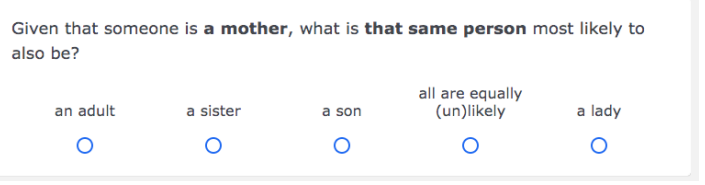

(b)

Figure 2: A) Example of a semantic differential question used to measure beliefs about identities (here, for the identity "thug" on the Evaluation dimension); B) Example of a hypothetical identity labeling question used to evaluate the importance of different dimensions.

sponses for four different, randomly selected identities. Each identity was given to a total of 15 different respondents. For each identity, we asked a set of seven questions, some of which had multiple subparts. Following prior work, beliefs for affective dimensions were solicited using a sliderbased Likert scale. For the Evaluative dimension, the opposing ends of the Likert scale were labeled "bad, awful" and "good, nice". For the Potency dimension, "powerless, little" and "powerful, big", and for the Activity dimension, "slow, quiet, inactive" and "fast, noisy, active". See Heise (2010) for more details on the development of these questions. The fourth and fifth question used Likert scales to measure beliefs about age and gender, with ends representing "young" and "old" and "Always male" and "Always female," respectively.

The sixth question asked "Of all [some identity, e.g., bullies], what percentage of them do you think are..." and then provided one slider each for the following ethnic/racial categories drawn from the planned 2020 Census: White, Hispanic or Latino, Asian, Middle Eastern, and Black. The seventh question, modeled after the association-based measures from Hill et al. (2015), asked "To what extent does thinking about [some identity, e.g., bullies] lead you to think about..." and then provided a slider for the following institutional settings: family, politics, (criminal) justice, medicine, business, education, and religion. Each slider had qualitative labels ranging from "Not at all", to "Somewhat", to "Immediate response". 


\begin{tabular}{ll} 
Dimension & Identities \\
\hline Politics & $\begin{array}{l}\text { conservative, Democrat, liberal, Repub- } \\
\text { lican, politician, senator }\end{array}$ \\
\hline Family & $\begin{array}{l}\text { brother, sister, daughter, son, father, } \\
\text { mother }\end{array}$ \\
\hline Law & $\begin{array}{l}\text { judge, criminal, lawyer, witness, cop, } \\
\text { police officer }\end{array}$ \\
\hline Medicine & $\begin{array}{l}\text { doctor, physician, surgeon, nurse, pa- } \\
\text { tient, dentist }\end{array}$ \\
\hline Business & $\begin{array}{l}\text { executive, consultant, secretary, intern, } \\
\text { banker, boss }\end{array}$ \\
\hline Gender & woman, guy, girl, boy, man, lady \\
\hline Age & $\begin{array}{l}\text { teenager, kid, child, toddler, adult, mi- } \\
\text { nor }\end{array}$ \\
\hline Race \& & $\begin{array}{l}\text { black, white, Hispanic, Asian, Arab, } \\
\text { American }\end{array}$ \\
Ethnicity & thug, idiot, jerk, goon, punk, bully \\
\hline $\begin{array}{l}\text { Negative } \\
\text { Evalua- } \\
\text { tion }\end{array}$ & \\
\hline Random & principal, scientist, coach \\
\hline
\end{tabular}

Table 1: The 57 identities we collect data on. Note that the dimensions used for sampling do not include all dimensions used in our belief measurement study.

\subsection{Identity Labeling Data}

We collect responses from 402 participants to a pair of identity labeling tasks. ${ }^{4}$ Note that these respondents are different than those who provided the belief measurements. Each participant answered a set of 40 hypothetical identity labeling questions. Questions could be either an IsA or a SeenWith question. An example of an IsA question is given in Figure 1B). SeenWith questions were formatted in the same way, except the question text instead says "Who would you say is most likely to be seen with a [mother]?"

Questions varied on both the identity provided in the text and the identities serving as potential answers. From the 57 identities we study, we create survey questions roughly ${ }^{5}$ as follows: for a given identity, we generate 14 random sets of the 56 other identities; each set contains four identities. We then generate one IsA and one SeenWith question for each of these sets, where these four identities constitute the possible answers to the question, and the given identity is used in the question text. This process is then repeated ten times for each identity. This process generates ten questions for each of the 3,192 identity pairs for each type of question.

\footnotetext{
${ }^{4}$ These identity labeling questions are similar to, but distinct from, those used in our prior work focused on the impact of semantic associations and semantic similarity on identity labeling decisions (Joseph and Carley, 2016).

${ }^{5}$ Due to a bug in Qualtrics, a small percentage of questions were not asked or asked more than once. See Appendix for details
}

\begin{tabular}{|c|c|}
\hline Variable & Description \\
\hline$i$ & A social identity (e.g. doctor, author) \\
\hline$d$ & A dimension of meaning (e.g. gender) \\
\hline$r$ & A survey respondent \\
\hline$S_{d, i, r}$ & $\begin{array}{l}\text { A matrix of survey responses to semantic } \\
\text { differential measures on a given dimension } \\
d \text { for identity } i \text { by respondent } r \text {. Each ob- } \\
\text { servation is in }[0,1] \text {, where } 0 \text { and } 1 \text { imply } \\
\text { something unique for each dimension de- } \\
\text { pending on the ends of the semantic differ- } \\
\text { ential scale. }\end{array}$ \\
\hline$\overline{\overline{S_{d, i, *}}}$ & $\begin{array}{l}\text { The average belief of identity } i \text { on dimen- } \\
\text { sion } d \text { in the survey data }\end{array}$ \\
\hline$E$ & $\begin{array}{l}\text { A matrix of word embeddings generated } \\
\text { from a particular combination of corpus } \\
\text { and embedding algorithm. We refer to this } \\
\text { as an embedding model }\end{array}$ \\
\hline$d w$ & $\begin{array}{l}\text { A dimension-inducing word set; that is, } \\
\text { a set of words that define the ends of a } \\
\text { particular dimension of meaning }\end{array}$ \\
\hline$w p$ & $\begin{array}{l}\text { A word position measurement model, i.e., } \\
\text { a method to place a given identity on a } \\
\text { given dimension of social meaning. }\end{array}$ \\
\hline$m_{E, d w, w p}(i)$ & $\begin{array}{l}\text { An embedding-based measurement model. } \\
\text { Defined by an embedding model } E \text {, a } \\
\text { dimension-inducing word set } d w \text {, and a } \\
\text { word position measurement model } w p \text {. Re- } \\
\text { turns a position for } i \text { along the induced } \\
\text { dimension }\end{array}$ \\
\hline
\end{tabular}

Table 2: Notation used in outlining our approach.

\subsection{Belief Measures From Prior Work}

To further substantiate our claims, we ensure our main results hold using three other datasets on beliefs about identities: beliefs about gender for 287 occupational identities from Bolukbasi et al. (2016), beliefs about 195 national and occupational identities on the Big Five Personality Traits from Agarwal et al. (2019), and beliefs about 654 identities on the Evaluation, Potency, and Activity dimensions by Smith-Lovin and Robinson (2015).

\section{Methods}

Our primary research question is, how accurately can we recover beliefs measured using surveys with word-embedding based measures? We study this first at the dimension level, i.e., how accurately do embedding-based measures reflect survey data across $a$ set of identities on a given dimension of social meaning? We then study accuracy at the $b e$ lief level, i.e., how accurately do embedding-based measures reflect survey data for specific identities on specific dimensions? Our approach is straightforward, but is best explained by introducing some additional notation, provided in Table 2 . 


\subsection{Dimension-level analysis}

At the dimension level, we consider first how different factors relating to the measurement itself impact accuracy. We then study why measurements are more accurate for some dimensions than others. We do so by connecting the degree of accuracy for a given dimension to how important that dimension is in how survey respondents select identities for others in our identity labeling task.

\subsubsection{Impact of measurement strategy}

As discussed above, the accuracy of embeddingbased measurements may vary across properties of the dimension being measured, as well as the way in which the embedding-based measurement is constructed. We first study the relative effects of a) the dimension $(d)$, b) the embedding model $(E), \mathrm{c})$ the dimension-inducing wordset $(d w)$, and d) the word position measurement model $(w p)$ on the accuracy of embedding-based measurements. As is standard in the literature, we use the Pearson correlation between the mean survey response and the output of the embedding-based measure as our definition of accuracy. That is, for a given dimension $d$, survey dataset $S$, embedding-based measure $m_{E, d w, w p}$, and set of identities of size $I$, we compute the accuracy of the embedding-based measure as the Pearson correlation between $\left\{\overline{S_{d, i_{0}, *}}, \overline{S_{d, i_{1}, *}}, \ldots, \overline{S_{d, i_{I}, *}}\right\}$ and $\left\{m_{E, d w, w p}\left(i_{0}\right), m_{E, d w, w p}\left(i_{0}\right), \ldots, m_{E, d w, w p}\left(i_{I}\right)\right\}$. We then run a linear regression to understand how accuracy varies across the factors considered.

Our analysis involves all dimensions of social meaning studied in the four survey datasets described above. For embedding models, $E$, we consider twelve different publicly available corpus/embedding combinations from prior work. To construct dimension-inducing wordsets, $d w$, we using one of three approaches. The first is to use the same terms as were placed on the semantic differential scale on the survey (e.g. powerless, powerful, little, big for Potency, as in Figure 2a). In certain cases, we also include a survey-augmented condition that extends this wordset using a thesaurus, after discussion amongst authors. Third, where applicable, we use direction-inducing wordsets from prior work. Finally, we consider several of the major established approaches in the literature for word position measurement $w p$. We use the approaches from Kozlowski et al. (2019), Swinger et al. (2019), Ethayarajh et al. (2019), Bolukbasi et al. (2016), and Garg et al. (2018). In the Appendix, we provide full details on the different values of $E, d w$, and $w p$ that we consider.

\subsubsection{Explaining variation across dimensions}

As we will show, controlling for $E, d w$ and $w p$, there are large differences in accuracy across dimensions. To better understand these differences across dimension, we compute two measurements. First, Kozlowski et al. (2019) show that the variance of the survey data on a dimension, that is, $\operatorname{Var}\left(\overline{S_{d, i_{0}, *}}, \overline{S_{d, i_{1}, *}}, \ldots, \overline{S_{d, i_{n}, *}}\right)$, is strongly correlated with the accuracy of embedding-based measures. However, they also note that "high explained variance... reveals little about how these valences are deployed in social life" (pg. 930). Here, we therefore compute a second measure that connects variance of the survey data on a given dimension to a significant social outcome, how strongly people rely on that dimension when labeling other people.

To do so, we first construct a $57 x 17$ matrix $X$ of scaled-and-centered mean survey responses for each identity on each dimension in our survey data, i.e. $X_{i_{0}, d_{0}}=\overline{S_{d_{0}, i_{0}, *}}$. We then construct an observation with a binary outcome that pairs the identity in the question with each possible answer. The outcome is 1 if the answer was selected, and 0 otherwise. For example, in Figure 2B), the pairings created would be "mother, adult", "mother, sister", "mother, son", and "mother, lady". If the respondent answered "lady", then the outcomes would be $0,0,0$, and 1 , respectively. The $40.3 \%$ of questions where respondents answered "all are equally unlikely" were ignored. In total, we obtained 9,597 responses where the respondent did not answer "All are equally (un)likely," split roughly evenly between SeenWith and IsA questions.

We then train a logistic regression model for IsA and SeenWith questions separately, each with seventeen parameters. For a given observation, the parameters represent the absolute difference between each dimension, e.g. the first parameter is $\left|X_{i_{q}, d_{0}}-X_{i_{a}, d_{0}}\right|$, where $i_{q}$ is "mother" in Figure $2 \mathrm{~B}), i_{a}$ is, e.g., "adult", and $d_{0}$ is, e.g., gender.

In the Appendix, we provide full results for these regressions. Intuitively, larger negative coefficients for a given dimension indicate that the further away two identities are on that dimension, the less likely the respondent is to select them as a pair. For example, we find that Evaluation has a strong negative correlation for IsA questions, indicating that respondents typically do not expect two identities to 
be assigned to the same person if one identity is perceived to be for "good people" and the other for "bad people". Positive coefficients imply assortativity on the dimension. For example, for SeenWith questions, Potency has a positive coefficient, implying that we expect powerful identities to be seen with less powerful counterparts. The magnitude of these coefficients represent the importance given to that dimension by survey respondents. We use the maximum of the two coefficients across SeenWith and IsA questions as a measure of this importance.

\subsection{Belief-level analysis}

We are also interested in accuracy for specific beliefs. For example, how accurately do embeddingbased measures reflect survey data on beliefs about the typical age of a boy? As an outcome for this belief-level analysis, we use a ranking task similar to prior work (Spirling and Rodriguez, 2019; Kozlowski et al., 2019). We describe this outcome by continuing with the example of beliefs about the age of boys. We first compute the set of identities $N$, for which $\overline{S_{a g e, b o y, *}}-\operatorname{se}\left(S_{a g e, b o y, *}\right)>\overline{S_{a g e, i, *}}+$ $\operatorname{se}\left(S_{a g e, i, *}\right)$, where $s e$ is the standard error function. That is, $N$ represents all identities we are reasonably confident respondents believed to be younger than boys. We then determine the subset of $N, N_{c}$, where boy is also ranked above those identities in the embedding measure. We do the same for identities survey respondents said were older than boys, adding these to $N$, and to $N_{c}$ if they are correctly ranked in the embedding measure. Finally, we use $\frac{N_{c}}{N}$ to study accuracy at the belief level.

We are interested both in overall levels of accuracy for belief-level measurements, as well as the factors that explain variation in accuracy. We consider four factors that might explain this variation (continuing with the age/boy example): $s d\left(S_{a g e, b o y, *}\right)$, the distance of $\overline{S_{a g e, b o y, *}}$ to the median over all identities on that dimension, the logged frequency of the identity in a large corpora, ${ }^{6}$ and the number of synsets for the identity in WordNet. To study the impact of these different factors, we use a generalized additive model with a binomial link function where $\frac{N_{c}}{N}$ is the outcome and points are weighted by $N$. Finally, as opposed to considering results across all possible $E, d w$, and $w p$, we first select those settings that maximize the Pearson correlation for each dimension.

\footnotetext{
${ }^{6}$ according to (Speer et al., 2018)
}

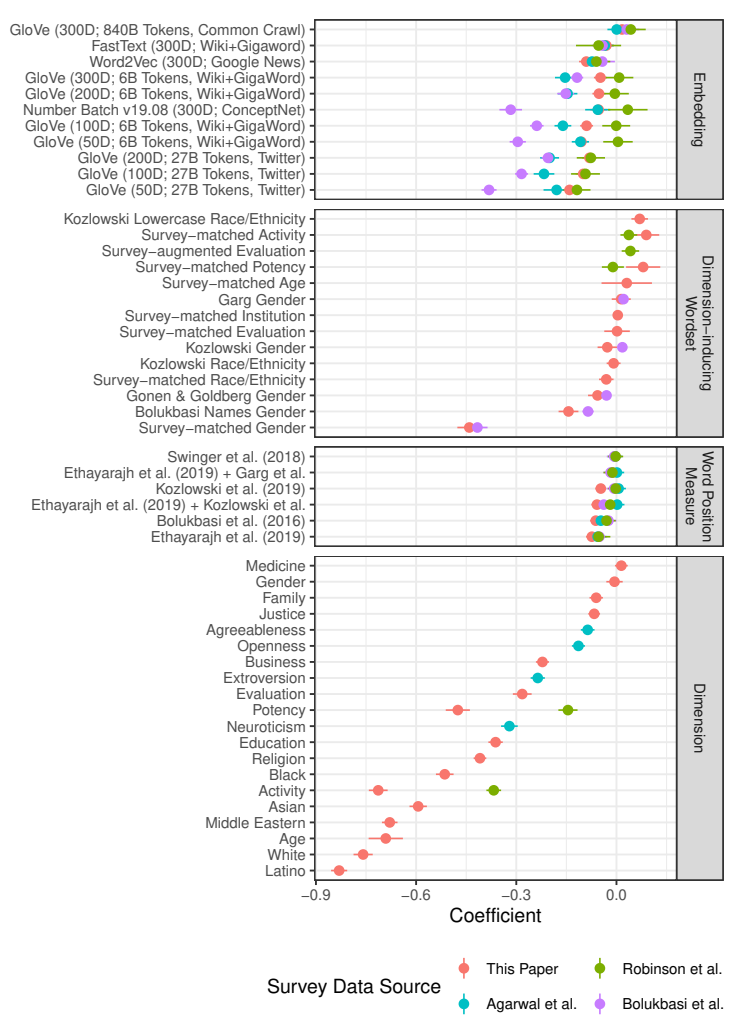

Figure 3: Regression results for the dimension-level analysis. Coefficients for each factor are relevant to a baseline. For the embedding models, the baseline is the FastText 300 dimensional model trained on the Common Crawl. For the dimension-inducing wordset, it is the terms used to define gender by Bolukbasi et al. (2016). For word position measurement, it is the approach from Garg et al. (2018), and for dimension, association with Politics.

\section{Results}

\subsection{Dimension-level results}

Across all conditions and survey datasets, the Pearson correlation between the embedding and survey measures is $0.32[.31, .33]$. However, considerable variation exists. Figure 3 presents results of a regression that attempts to explain the sources of this variance (x-axis) and the effects of each source (yaxis). Separate colors represent results from the four different survey datasets analyzed. In general, results are largely consistent across the different datasets, and thus we will not emphasize differences across datasets below.

Figure 3 shows that the embedding model used can decrease correlation by as much as . 35 . As others have found, this effect decreases when one focuses only on 300-dimensional embeddings. It is worth noting, however, that no embedding model is universally best. For example, nine of the twelve 
embedding models studied are responsible for producing the highest observed correlation for at least one dimension.

Selection of the dimension-inducing words, $d w$, also has a limited effect. The one exception is when survey-matched words are used for the Gender dimension, where correlations drop by, on average, around 0.5 relative to the "he/she" baseline. The fact that using the same words as the semantic differential scale is a terrible choice, but for only one of the seventeen dimensions studied, reflects the fact that selection of $d w$, like elements of other forms of quantitative social science, remains a mix of art and science (Sterling and Taveter, 2009).

In contrast, even the most scientifically appealing approaches to word position measurement (Ethayarajh et al., 2019) provide marginal gains. The only consistent observation we draw is that approaches that normalize measurements across dimensions related to the same overarching concept (e.g. that normalize racialized beliefs across all perceived dimensions of race) perform slightly better. Results thus reflect that the details of measurement are less important than what is being measured.

Reflecting this same fact, the strongest impacts on correlation between the survey and embeddingbased measures come from which dimension is being studied. Some of these results reflect the salience of these dimensions in social life. Associations to institutions, which are most accurately measured on average, are a primary tool we use to sort people into groups (MacKinnon and Heise, 2010). And stronger correlations between the embedding and survey-based measures for Evaluation and Potency, relative to Activity, reflects the increased importance in affective perceptions of these two dimensions (Rogers et al., 2013). However, scholars largely agree that trait-based beliefs on gender and race serve as "default characteristics" (Ridgeway and Smith-Lovin, 1999) along which we almost automatically categorize others (Todorov et al., 2015). Given their shared salience, why is gender the only trait that can be accurately measured?

Figure 4A) shows, as first identified by Kozlowski et al. (2019), that much of this is due to the variance of the survey data along that dimension; the correlation between variance and the coefficients in Figure 3 is 0.91. However, as discussed above, Kozlowski et al. (2019) study more general concepts on more general dimensions, and note that they have no easy way to connect their

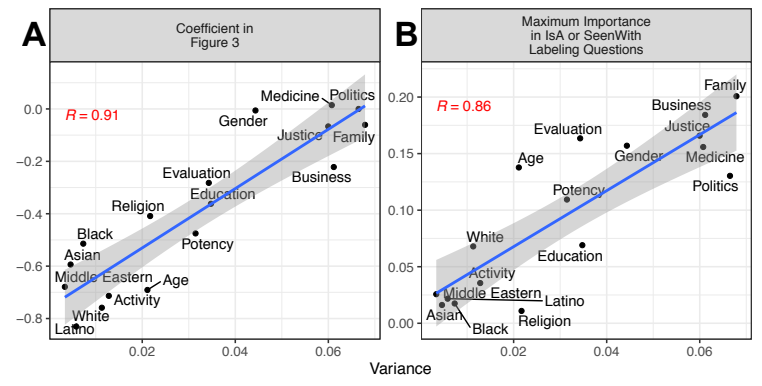

Figure 4: In both A) and B), the y-axis gives the coefficient value for the regression presented in Figure 3. In A), the $x$-axis represents the variance in survey means along the dimension. In B), an estimate of the dimension's importance for identity labeling
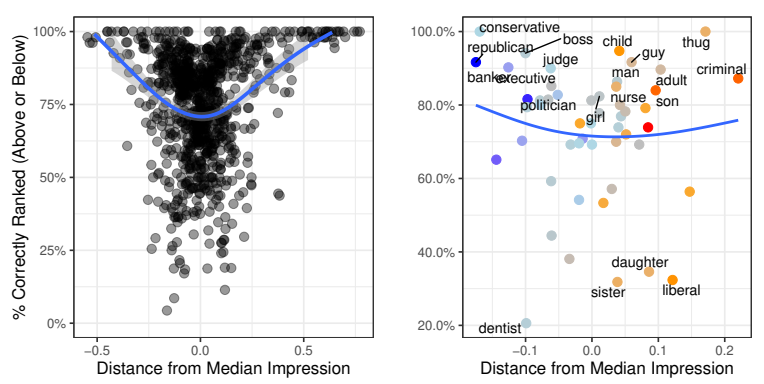

Figure 5: Results for our belief-level outcome (y-axis) as a belief's distance from the dimension-level median (x-axis) varies for all dimensions (left) and only the "Black" dimension (right). Points on the right are colored by variance, with higher (lower) variance estimates colored blue (red)

observations to any critical social processes. In contrast, here, Figure 4B) shows a significant positive correlation between variance in the survey data along a dimension (and hence measurement accuracy) and that dimensions' importance in explaining patterns of labeling in our identity labeling task. Embedding-based measures of beliefs about identities, we therefore show, are most likely to reflect traditional survey measures particularly when those beliefs are salient for identity labeling.

Critically, then, results for biases in word embeddings are tied not only to the salience of dimensions in general social life, but also to the identities selected for measurement. Selecting only heavily racialized and non-gendered identities, for example, might well reverse the positions of racialized dimensions and gender in Figure 4. This makes it all the more critical to identify theoretically-driven concepts - salience in labeling, and variance in measurement - that move beyond measures of specific identities on specific dimensions to help us understand what is measurable and what is not, 
particularly when survey data is not available.

\subsection{Belief-level results}

As with the dimension-level results, we find that embedding-based measures are generally accurate predictors of survey-based measures for specific beliefs. On average, $74.9 \%$ of the beliefs collected for this paper are correctly ranked, as are $82.1 \%$, $72.0 \%$, and $71.4 \%$ of the beliefs from Bolukbasi et al. (2016), Smith-Lovin and Robinson (2015), and Agarwal et al. (2019), respectively. One caveat to keep in mind, however, is that we focus only on the single best embedding measurement approach for each source/dimension combination.

Regardless, as with the dimension-level results, there is considerable variance at the belief level. Some of this variance (approximately $32 \%$, see the Appendix for full regression results ) can be explained by the factors we consider. The strongest explanation we find to explain ranking accuracy, reflected in the left-hand plot in Figure 5, is the distance of the survey-based belief measure from the median on its dimension. At the extremes, ranking accuracy is almost perfect. Because extreme observations are also most likely to be low variance-for example, consider that beliefs at the most extreme values of a scale must have zero variance-a more general claim can be made: word embedding-based measures accurately capture our most extreme and agree-upon beliefs about people, but show significant unexplained (at least by us) variance for more neutral and/or less-agreed upon beliefs.

This variance is on display in the right-hand plot in Figure 5, which gives results for the blackness dimension. The embedding-based measure captures with perfect accuracy racialized perceptions of the identities thug and criminal, but not, e.g., liberal, which is similar along the other explanatory factors we consider here. As far as we are aware, it remains an open question as to why this is the case.

\section{Conclusion}

In this paper, we asked, can we trust measures of beliefs about people derived from word embeddings? We find the answer to be yes, at least on average. Depending on one's perspective, this could be good or bad. From a cultural studies/social psychological perspective, this positive correlation further validates efforts to use word embeddings to study perceptions of people historically, at scale, and in context. On the other hand, from the "bias" per- spective, this suggests that a vast array of social biases are encoded in embeddings.

However, we also find that some beliefs- specifically, extreme beliefs on salient dimensions - are easier to measure than others. More generally, across four datasets, we find that what we measure is more important than how we measure it. Again, two different perspectives on this are needed. With respect to the study of culture and human stereotypes, we may be safest in studying only the most extreme results from embedding models, as has been done by, e.g., Spirling and Rodriguez (2019).

From the bias perspective, given the rash of recent work on debiasing word embeddings, our results suggest that much more attention needs to be paid to how we are evaluating these approaches. Currently, upstream evaluations of debiasing are centered almost exclusively on occupational identities on gender, where some of the most salient social biases we know of exist (Ridgeway, 2011). Others have argued that removing these salient beliefs may not remove gender information from embeddings (Gonen and Goldberg, 2019). But Gonen and Goldberg's 2019 argument relies on a technical deficiency of existing approaches. We can make a similar critique by simply changing what is being measured. For example, the correlation between gender beliefs and the gender direction in the HardDebiased embeddings of Bolukbasi et al. (2016) is $0.05(\mathrm{p}=.84)$ using identities in their data, and 0.4 $(p<.05)$ using the identities in our data.

Similarly, removing gender bias does not remove bias on other dimensions. For example, while Sweeney and Najafian (2019) show that the NumberBatch embeddings harbor the least gender bias, we find that they are the only embedding to show consistently high correlations with age, leading to the potential for ageism downstream. More generally, stereotypes exist along a network of beliefs (Freeman and Ambady, 2011) reflecting unwarranted correlations between many dimensions (Ridgeway, 2011); we must therefore be careful not to expect that removing meaning along one dimension will expel social biases from our models.

\section{Acknowledgements}

K.J. was supported by NSF IIS-1939579. This research was supported in part a SUNY Germination Space Grant. We thank Lynn Smith-Lovin, Lisa Friedland, Tobias Schroeder, and Yuhao Du for comments on earlier versions of this work. 


\section{References}

Oshin Agarwal, Funda Durupınar, Norman I. Badler, and Ani Nenkova. 2019. Word embeddings (also) encode human personality stereotypes. In Proceedings of the Eighth Joint Conference on Lexical and Computational Semantics (*SEM 2019), pages 205211, Minneapolis, Minnesota. Association for Computational Linguistics.

Jisun An, Haewoon Kwak, and Yong-Yeol Ahn. 2018. Semaxis: A lightweight framework to characterize domain-specific word semantics beyond sentiment. In $A C L$.

William H. Batchelder and A. Kimball Romney. 1988. Test theory without an answer key. Psychometrika, 53(1):71-92.

Joseph Berger, Robert Z. Norman, James W. Balkwell, and Roy F. Smith. 1992. Status inconsistency in task situations: A test of four status processing principles. American Sociological Review, pages 843-855.

Tolga Bolukbasi, Kai-Wei Chang, James Zou, Venkatesh Saligrama, and Adam Kalai. 2016. Man is to Computer Programmer as Woman is to Homemaker? Debiasing Word Embeddings. arXiv preprint arXiv: 1607.06520.

Marc-Etienne Brunet, Colleen Alkalay-Houlihan, Ashton Anderson, and Richard Zemel. 2019. Understanding the Origins of Bias in Word Embeddings. In International Conference on Machine Learning, pages $803-811$.

Aylin Caliskan, Joanna J. Bryson, and Arvind Narayanan. 2017. Semantics derived automatically from language corpora contain human-like biases. Science, 356(6334):183-186.

Kaytlin Chaloner and Alfredo Maldonado. 2019. Measuring Gender Bias in Word Embeddings across Domains and Discovering New Gender Bias Word Categories. In Proceedings of the First Workshop on Gender Bias in Natural Language Processing, pages 25-32, Florence, Italy. Association for Computational Linguistics.

Maria De-Arteaga, Alexey Romanov, Hanna Wallach, Jennifer Chayes, Christian Borgs, Alexandra Chouldechova, Sahin Geyik, Krishnaram Kenthapadi, and Adam Tauman Kalai. 2019. Bias in bios: A case study of semantic representation bias in a high-stakes setting. In Proceedings of the Conference on Fairness, Accountability, and Transparency, pages $120-128$.

Dawn Marie Dow. 2016. The deadly challenges of raising African American boys: Navigating the controlling image of the "thug". Gender \& Society, 30(2):161-188.

Kawin Ethayarajh, David Duvenaud, and Graeme Hirst. 2019. Understanding undesirable word embedding associations. In Proceedings of the 57th Annual
Meeting of the Association for Computational Linguistics, pages 1696-1705, Florence, Italy. Association for Computational Linguistics.

Ethan Fast, Tina Vachovsky, and Michael S. Bernstein. 2016. Shirtless and dangerous: Quantifying linguistic signals of gender bias in an online fiction writing community. In Tenth International AAAI Conference on Web and Social Media.

Anjalie Field, Gayatri Bhat, and Yulia Tsvetkov. 2019. Contextual affective analysis: A case study of people portrayals in online\# metoo stories. In Proceedings of the International AAAI Conference on Web and Social Media, volume 13, pages 158-169.

Susan T. Fiske, Amy JC Cuddy, Peter Glick, and Jun $\mathrm{Xu}$. 2002. A model of (often mixed) stereotype content: Competence and warmth respectively follow from perceived status and competition. Journal of personality and social psychology, 82(6):878.

Jonathan B. Freeman and Nalini Ambady. 2011. A dynamic interactive theory of person construal. Psychological review, 118(2):247.

Nikhil Garg, Londa Schiebinger, Dan Jurafsky, and James Zou. 2018. Word embeddings quantify 100 years of gender and ethnic stereotypes. Proceedings of the National Academy of Sciences, 115(16):E3635-E3644.

Hila Gonen and Yoav Goldberg. 2019. Lipstick on a pig: Debiasing methods cover up systematic gender biases in word embeddings but do not remove them. In Proceedings of NAACL-HLT, pages 609-614.

David R. Heise. 1987. Affect control theory: Concepts and model. The Journal of Mathematical Sociology, 13(1-2):1-33.

David R. Heise. 2007. Expressive Order. Springer.

David R. Heise. 2010. Surveying Cultures: Discovering Shared Conceptions and Sentiments. John Wiley \& Sons.

Felix Hill, Roi Reichart, and Anna Korhonen. 2015. Simlex-999: Evaluating semantic models with (genuine) similarity estimation. Computational Linguistics, 41(4):665-695.

Alexander Hoyle, Wolf-Sonkin, Hanna Wallach, Isabelle Augenstein, and Ryan Cotterell. 2019. Unsupervised discovery of gendered language through latent-variable modeling.

Kenneth Joseph and Kathleen M. Carley. 2016. Relating semantic similarity and semantic association to how humans label other people. NLP+CSS 2016, page 1.

Kenneth Joseph, Wei Wei, and Kathleen M. Carley. 2016. Exploring patterns of identity usage in tweets: A new problem, solution and case study. In Proceedings of the 25th International Conference on World Wide Web, pages 401-412. 
Kenneth Joseph, Wei Wei, and Kathleen M. Carley. 2017. Girls rule, boys drool: Extracting semantic and affective stereotypes from Twitter. In 2017 ACM Conference on Computer Supported Cooperative Work. $(C S C W)$.

George Karabatsos and William H. Batchelder. 2003. Markov chain estimation for test theory without an answer key. Psychometrika, 68(3):373-389.

Austin C. Kozlowski, Matt Taddy, and James A. Evans. 2019. The Geometry of Culture: Analyzing the Meanings of Class through Word Embeddings. American Sociological Review, 84(5):905-949.

Anne Lauscher and Goran Glavaš. 2019. Are we consistently biased? multidimensional analysis of biases in distributional word vectors. In Proceedings of the Eighth Joint Conference on Lexical and Computational Semantics (* SEM 2019), pages 85-91.

Neil J. MacKinnon and David R. Heise. 2010. Self, Identity, and Social Institutions. Palgrave Macmillan.

Otto H MacLin and Roy S Malpass. 2001. Racial categorization of faces: The ambiguous race face effect. Psychology, Public Policy, and Law, 7(1):98.

George J. McCall and Jerry L. Simmons. 1978. Identities and interactions. New York.

Tomas Mikolov, Kai Chen, Greg Corrado, and Jeffrey Dean. 2013. Efficient estimation of word representations in vector space. arXiv preprint arXiv:1301.3781.

Tomas Mikolov, Edouard Grave, Piotr Bojanowski, Christian Puhrsch, and Armand Joulin. 2018. Advances in pre-training distributed word representations. In Proceedings of the International Conference on Language Resources and Evaluation (LREC 2018).

Inom Mirzaev, Anthony Schulte, Michael Conover, and Sam Shah. 2019. Considerations for the interpretation of bias measures of word embeddings. arXiv preprint arXiv:1906.08379.

Charles Egerton Osgood, William H. May, Murray Samuel Miron, and Murray S. Miron. 1975. Cross-Cultural Universals of Affective Meaning, volume 1. University of Illinois Press.

Olutobi Owoputi, Brendan O'Connor, Chris Dyer, Kevin Gimpel, Nathan Schneider, and Noah A Smith. 2013. Improved part-of-speech tagging for online conversational text with word clusters. In Proceedings of NAACL.

Jeffrey Pennington, Richard Socher, and Christopher D. Manning. 2014. Glove: Global vectors for word representation. In Empirical Methods in Natural Language Processing (EMNLP), pages 1532-1543.
Cecilia L. Ridgeway. 2011. Framed by Gender: How Gender Inequality Persists in the Modern World. Oxford University Press.

Cecilia L. Ridgeway and Lynn Smith-Lovin. 1999. The gender system and interaction. Annual review of sociology, 25(1):191-216.

Kimberly B. Rogers, Tobias Schröder, and Wolfgang Scholl. 2013. The Affective Structure of Stereotype Content Behavior and Emotion in Intergroup Context. Social Psychology Quarterly, 76(2):125-150.

L. Smith-Lovin and W. Douglas. 1992. An affect control analysis of two religious subcultures. Social perspectives on emotion, 1:217-47.

L. Smith-Lovin and Dawn T. Robinson. 2015. Interpreting and Responding to Events in Arabic Culture. Final Report to Office of Naval Research, Grant N00014-09-1-0556.

Robyn Speer, Joshua Chin, and Catherine Havasi. 2017. ConceptNet 5.5: An open multilingual graph of general knowledge. pages 4444-4451.

Robyn Speer, Joshua Chin, Andrew Lin, Sara Jewett, and Lance Nathan. 2018. Luminosoinsight/wordfreq: v2.2.

Arthur Spirling and P Rodriguez. 2019. Word embeddings: What works, what doesn't, and how to tell the difference for applied research. Technical report, Working paper.

Leon Sterling and Kuldar Taveter. 2009. The art of agent-oriented modeling. MIT press.

Chris Sweeney and Maryam Najafian. 2019. A Transparent Framework for Evaluating Unintended Demographic Bias in Word Embeddings. In Proceedings of the 57th Annual Meeting of the Association for Computational Linguistics, pages 1662-1667, Florence, Italy. Association for Computational Linguistics.

Nathaniel Swinger, Maria De-Arteaga, Neil Thomas Heffernan IV, Mark DM Leiserson, and Adam Tauman Kalai. 2019. What Are the Biases in My Word Embedding? In Proceedings of the 2019 AAAI/ACM Conference on AI, Ethics, and Society, AIES '19, pages 305-311, New York, NY, USA. ACM.

Alexander Todorov, Christopher Y. Olivola, Ron Dotsch, and Peter Mende-Siedlecki. 2015. Social Attributions from Faces: Determinants, Consequences, Accuracy, and Functional Significance. Annual Review of Psychology, 66(1):519-545.

Nicholas Winter, Tyler Burleigh, Ryan Kennedy, and Scott Clifford. 2019. A Simplified Protocol to Screen Out VPS and International Respondents Using Qualtrics. SSRN Scholarly Paper ID 3327274, Social Science Research Network, Rochester, NY. 


\section{A Embedding Models}

We use twelve publicly available embedding models. We use all public GloVe (Pennington et al., 2014) models ${ }^{7}$, FastText (Mikolov et al., 2018) models $^{8}$, the original Word2Vec (Mikolov et al., 2013) $\operatorname{model}^{9}$, and v19.08 of the NumberBatch (Speer et al., 2017) model. ${ }^{10}$

\section{B Word Position Measurement Models}

Table 3 outlines the word position measurement models used in the present work. The table provides information on the authors of the measure, whether or not embeddings are normalized before analysis, how words are measured once a direction has been specified, how a direction is specified, and whether or not the method is "multi-class," described further below.

Notationally, we have tried to remain as close to the original works as possible. Therefore, $w$ is the identity to be measured, and $b$ is the vector indicating the direction along which it is to be measured. For Garg et al. (2018), $b_{l}$ and $b_{r}$ represent words in the left-hand dimension-inducing word set (e.g. "man" and "him" for gender) and $b_{r}$ the right-hand of the dimension-inducing word sets (e.g. "woman" and "her" for gender). The variables $p_{i, l}$ and $p_{i, r}$ have similar meanings for Swinger et al. (2019) and Bolukbasi et al. (2016).

We use the approaches of Garg et al. (2018), Kozlowski et al. (2019), Ethayarajh et al. (2019), and Swinger et al. (2019) exactly as described in the original texts, except for one modification. In the case where a paired set of terms is requiredall cases except Garg et al. (2018) and Swinger et al. (2019) —and we have a multi-class measurement (e.g. we measure four different dimensions of racialized beliefs), we first identify a default dimension and then compare all other dimensions to it. For race, we follow theory on perceptions of default race categories and assign White to be the default race (MacLin and Malpass, 2001), and the comparison point for White, following Kozlowski et al. (2019), to be Black. For the associative dimensions, we select family for the default, and

\footnotetext{
${ }^{7}$ https://nlp.stanford.edu/projects/ glove/

${ }^{8}$ https: / / fasttext.cc/docs/en/ english-vectors.html

${ }^{9}$ https://code.google.com/archive/p/ word2vec/

${ }^{10}$ https://github.com/commonsense/ conceptnet-numberbatch
}

compare family to politics.

In addition, we consider the possibility that the computationally appealing approach from (Ethayarajh et al., 2019) may be improved by using a different direction specification approach. Therefore, we consider two additional word position measurement models, Ethayarajh et al. (2019) + Garg et al. (2018), and Ethayarajh et al. (2019) + Kozlowski et al. (2019), that are the same as the original model but using the direction specification method in these two papers instead of the method from Bolukbasi et al. (2016), as was done in the original paper.

\section{Further Details - Identity Selection for Our Survey Data}

In selecting identities for each cluster, we also ensured that the words selected were a) expressed most frequently as identities, b) were in a standard set of lexical resources and thus common English terms and c) were used relatively frequently. To ensure the identities were used first and foremost as an identity (and not, e.g., as a verb or place name), we first used both the NLP python library spacy and Wordnet to identify any identities for which the dominant sense was a verb (e.g. suspect, accused) and removed these from consideration. To ensure that the identities were in common lexical databases, we removed words which were not in Wordnet as a noun or an adjective. Finally, to ensure that identity words were used frequently, we checked that they were used frequently in either a fairly informal medium, Twitter, or in a fairly formal medium, Wikipedia. To check the former case, we use the frequency counts of words from 56M tweets given by Owoputi et al. (2013) and retain only those identities used in more than 2500 tweets. To check Wikipedia, we first extract all 532,051 "clean" pages from a Wikipedia dump from December, 2015. A clean page is a page that is not labeled as a stub, that was still active one month after the dump was created, and that also has more than 50 views over 2 year span, where we pull one random hour for each day.

\section{Further Details - Belief Measurement Data}

\section{D.1 Measurement}

The slider bar for the affective dimensions gives labels at different points, ranging from "Infinitely" to "Slightly" on both ends, with a "Neutral" option 


\begin{tabular}{|c|c|c|c|c|}
\hline Measure & Normalized? & Position Measure & Direction-Specification & Multiclass \\
\hline $\begin{array}{l}\text { Ethayarajh } \\
\text { et al. (2019) }\end{array}$ & $\mathrm{N}$ & $\frac{\langle w, b\rangle}{\|b\|}$ & Same as Bolukbasi et al. (2016) & $\mathrm{N}$ \\
\hline $\begin{array}{l}\text { Kozlowski } \\
\text { et al. (2019) }\end{array}$ & $\mathrm{Y}$ & $\frac{\langle w, b\rangle}{\|b\|\||| w\|}$ & $\sum_{p_{i} \in P} \frac{p_{i, l}-p_{i, r}}{\|P\|}$ & $\mathrm{N}$ \\
\hline $\begin{array}{l}\text { Bolukbasi } \\
\text { et al. (2016) }\end{array}$ & $\mathrm{Y}$ & $\frac{\langle w, b\rangle}{\|b\|\||| w\|}$ & $S V D\left(\mathrm{c}\left(p_{i, j}-\mu_{p_{i j}} \quad p_{i} \in P\right)\right)$ & $\mathrm{N}$ \\
\hline $\begin{array}{l}\text { Swinger et al. } \\
\text { (2019) }\end{array}$ & $\mathrm{Y}$ & $\begin{array}{l}\operatorname{avg}_{p_{i} \in P} \frac{\left\langle w, p_{i, l}\right\rangle}{\left\|w\left|\left\|\left|\left\|\mid i_{i, l}\right\|\right.\right.\right.\right.}- \\
\operatorname{avg}_{p_{i} \in P} \frac{\left\langle w, p_{i, r}\right\rangle}{\|w\|\left\|\mid p_{i, r}\right\|}\end{array}$ & N/A & $\mathrm{Y}$ \\
\hline $\begin{array}{l}\text { Garg et al. } \\
(2018)\end{array}$ & $\mathrm{Y}$ & $\left\|w-b_{r}\right\|-\left\|w-b_{l}\right\|$ & $\overline{b_{l}}:=\sum_{p_{i} \in p_{r}} \frac{p_{i}}{\|P\|}$ & $\mathrm{Y}$ \\
\hline
\end{tabular}

Table 3: Details of the prior work on word position measurement models from which we draw. We use each model listed here, as well as using the approach of Ethayarajh et al. (2019) but using direction specification as described by Garg et al. (2018) and Kozlowski et al. (2019).

in the middle. The age slider had the following qualitative labels, spaced equally across the slider bar: "Baby, Child, Teenager, 20s, 30s, 40s, 50s, $60 \mathrm{~s}, 70 \mathrm{~s}, 80 \mathrm{~s}, 90 \mathrm{~s},>=100$ ". The gender slider had the following labels, spaced equally across the slider: "Always Male, Mostly Male, Equally Male or Female, Mostly Female, Always Female".

For the race/ethnicity beliefs, order of the sliders was randomized, and the starting value for each was set to $20 \%$. With respect to discussions about the 2020 census, most importantly, demographers have pushed to include Hispanic or Latino as a racial category rather than to split it out into its own separate question.

For the associative belief question, presentation order was randomized. The form of the question is drawn from other studies seeking to elicit cognitive associations between a term and a set of other concepts, e.g. from Hill et al. (2015). The specific institutions were originally drawn from the clustering used to determine our identities. However, we added the education and religion institutions after determining they would be necessary for a more complete meaning space, as suggested by the institutional settings with which identities are commonly associated as discussed by MacKinnon and Heise (2010).

Finally, pilot testing suggested that respondents became confused when provided with certain identities that had meanings that were used to construct the question - for example, on the race question, respondents became confused when being asked "Of all white people, what percentage of them do you think are ... [White]?" We, therefore, removed the gender question from the identities guy, boy, girl, lady, man, and woman, and removed the race question from the identities Asian, White person, Black person, Arab, and Hispanic.

\section{D.2 Participant Sample}

In total, 252 Mechanical Turk workers completed the survey. These had greater than a $95 \%$ completion rate and had completed over 1,000 HITs. These workers also were located within the United States. In order to ensure this was the case, we leveraged a tool provided by Winter et al. (2019) to ensure that participants' IP addresses were located within the United States and that they were not using a VPN. We further ensured competency and attention by including two attention check questions. Five respondents were rejected because they failed attention checks. Sample demographics were not collected for Task 1, because we make the deliberate assumption that measurements from surveys of any individuals within a national culture can serve as the foundation for a meaning space. However, as noted, we do ensure cultural expertise by ensuring participants are native English speakers located in the U.S.

\section{D.3 Ethical Approval}

This study was approved by the Institutional Review board of the University at Buffalo.

\section{D.4 Summary Results}

Figure 6 provides $95 \%$ bootstrapped confidence intervals for the Evaluation, Potency, and Activity dimensions for each identity in the survey. The 
measurements in our survey are compared to results from Smith-Lovin and Robinson (2015). The vast majority of our estimates overlap closely with theirs, signifying the broad generality of the measurement tools used by ACT across individuals within a national culture. Where differences arise, we do not believe one dataset or the other appears to be universally more accurate, and further, given the number of comparisons (171, 3 per each of 57 identities), we should expect even by chance some larger differences in the measurements.

Figure 7 provides full results for associative meanings. The figure shows that in general, identities assumed to cluster within a particular institution were rated as having the highest associations with that institution. However, it is also clear that other identities were also strongly aligned with the various institutions in ways that did not follow exactly from the construction of our identity set. For example, the identity thug was included as a negative affect term, but was perceived to have a strong association to the judicial institution.

Figure 8 provides full results for the expected age and gender of each identity. Note that some identities with a denotative meaning aligned with these dimensions were included, because pilot tests did not suggest confusion for these identities. We therefore attempted to include as many identities as possible in the actual measurement.

Figure 9 provides full results for the race question we asked. All identities were assumed by participants to be more White than expected by chance. Recall that the racial question was not posed for the denotatively aligned racial identities we studied.

\section{E Further Details - Identity Labeling Task}

\section{E.1 Participant Sample}

We collected valid data from 402 Mechanical Turk workers were located in the United States, had greater than a $95 \%$ completion rate and had completed over 1,000 HITs. To assess accuracy for respondents, we randomly sampled 5 questions from each respondent and ensured that answers did not appear to be entered randomly. The sample's gender was $53.7 \%$ female, and $45.6 \%$ male (.7\% did not say). A total of $89.4 \%$ had at least some college or vocational training, $53.8 \%$ of the sample had completed at least a Bachelor's degree, and $16.1 \%$ had a post-graduate degree. Almost all $(96.7 \%)$ of the sample were born in and had lived between $75-100 \%$ of their life in the United States. With respect to age, $9.0 \%$ of the sample was aged $18-24,17.2 \%$ aged $25-29,33.3 \%$ aged $30-39$, and $40.0 \%$ aged 40 or older (.5\% did not say). Finally, the sample was largely white, $83.3 \%$ of participants were White or Caucasian.

\section{E.2 Ethical Approval}

The survey carried out was approved by the Institutional Review board of Carnegie Mellon University.

\section{E.3 Additional Implementation Details}

As noted in the text, and replicated here for clarity, from the 57 identities in Table 1, we create survey questions for the identity labeling task as follows: for a given identity, we generate 14 random sets of the 56 other identities; each set contains four identities. We then generate one IsA and one SeenWith question for each of these sets, where these four identities constitute the possible answers to the question, and the given identity is used in the question text. This process is then repeated ten times for each identity. This process generates exactly ten questions for each of the $3,192^{11}$ identity pairs for each type of question.

The intention was, therefore, to have exactly ten questions for each identity pair for each question type where the first identity in the pair is shown in the question and the second identity in the pair is shown as a possible answer. In each case, by construction, the other possible answers were randomly selected. Unfortunately, our survey suffered from a bug with the Qualtrics software used, where the option to present questions an even number of times fails in unclear cases. Due to this error, some of our identity pairs were not seen exactly ten times. Specifically, $3.4 \%$ were asked less than 6 times, $40 \%$ were asked less than ten times, and $40.4 \%$ were asked more than ten times. While this does not affect our analyses, because they do not rely on any exact number of questions per identity pairing, it is important to note for purposes of any future work with the dataset.

Such issues aside, the process described generated 15,960 questions. These questions produced a total of 16,080 responses (a small number of questions-120, or $0.7 \%$ - were asked more than once in attempts to address the Qualtrics bug) that were split evenly, 40 questions per respondent.

\footnotetext{
${ }^{11} 57 * 56=3,192$
} 


\section{E.4 Results}

Figure 10 presents full results for the regression models described in the text to identify the importance of each dimension for identity labeling.

\section{F Additional Details - RQ2}

Figures 11-14 present results from generalized additive models with the four dependent variables described in the main text on the rank-level outcome variable at the belief level. The models explain $31.8 \%, 34.2 \%, 16.9 \%$, and $21.6 \%$ of the deviance for the data from this paper, Bolukbasi et al. (2016), Agarwal et al. (2019), and Smith-Lovin and Robinson (2015), respectively. Across the four datasets, the only consistent predictor is the distance of the survey-based belief measure from the median. Note, however, that in the data from SmithLovin and Robinson (2015), this pattern does not hold at the extremes. Further analyses suggests this is due to a small number of outliers on the extremes of the Evaluation dimension, and does not appear to reflect any interesting trend worth additional consideration. Additionally, we note that, the authors of Bolukbasi et al. (2016) could only provide us with a mean per-identity estimate, and thus no information on the variance of those estimates is used in our results. 


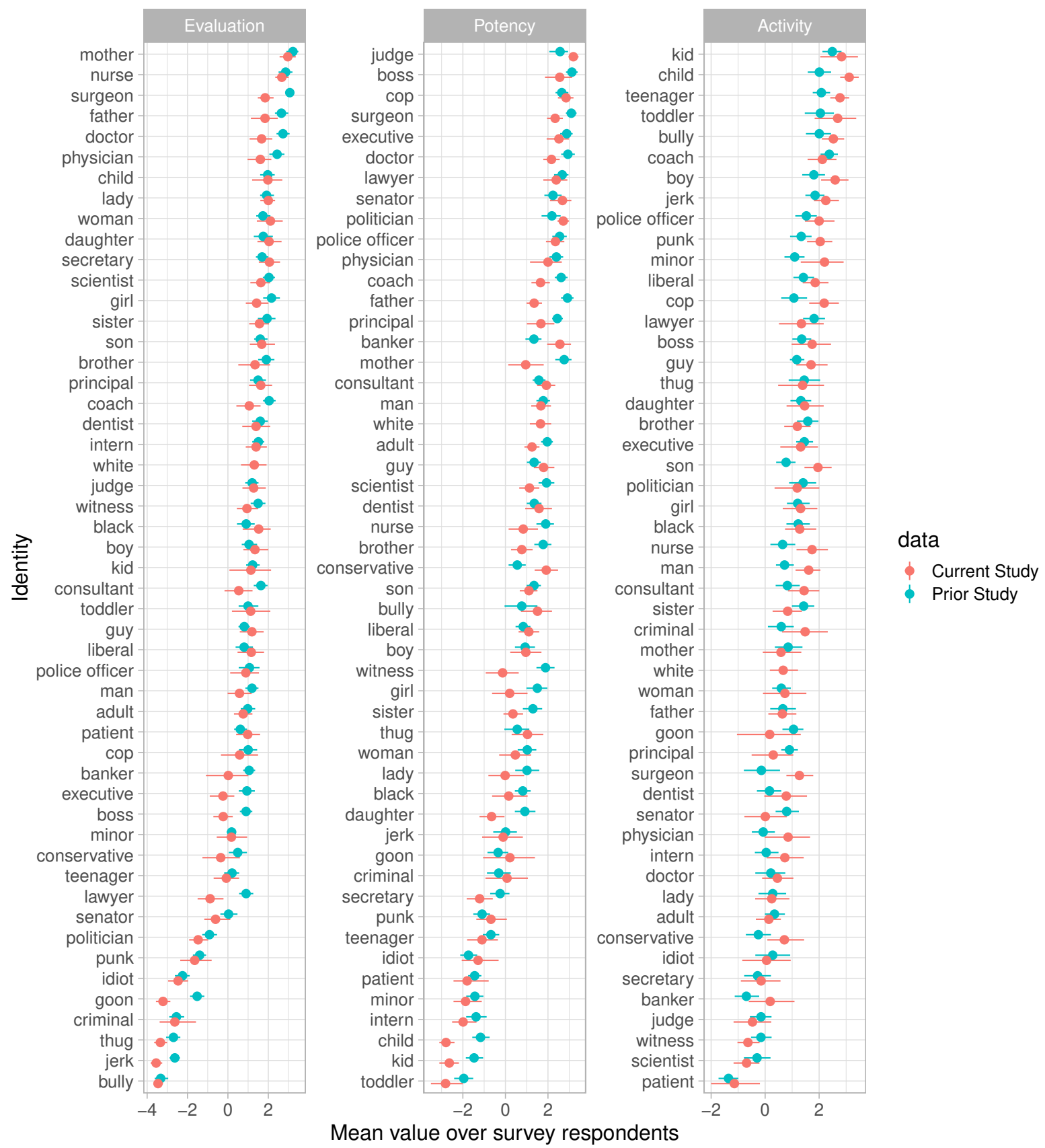

Figure 6: 95\% bootstrapped confidence intervals for estimates of the Evaluation, Potency, and Activity dimensions. Results from the current study are in red, results from a prior study from Smith-Lovin and Robinson (2015) are given for comparison in cyan. 

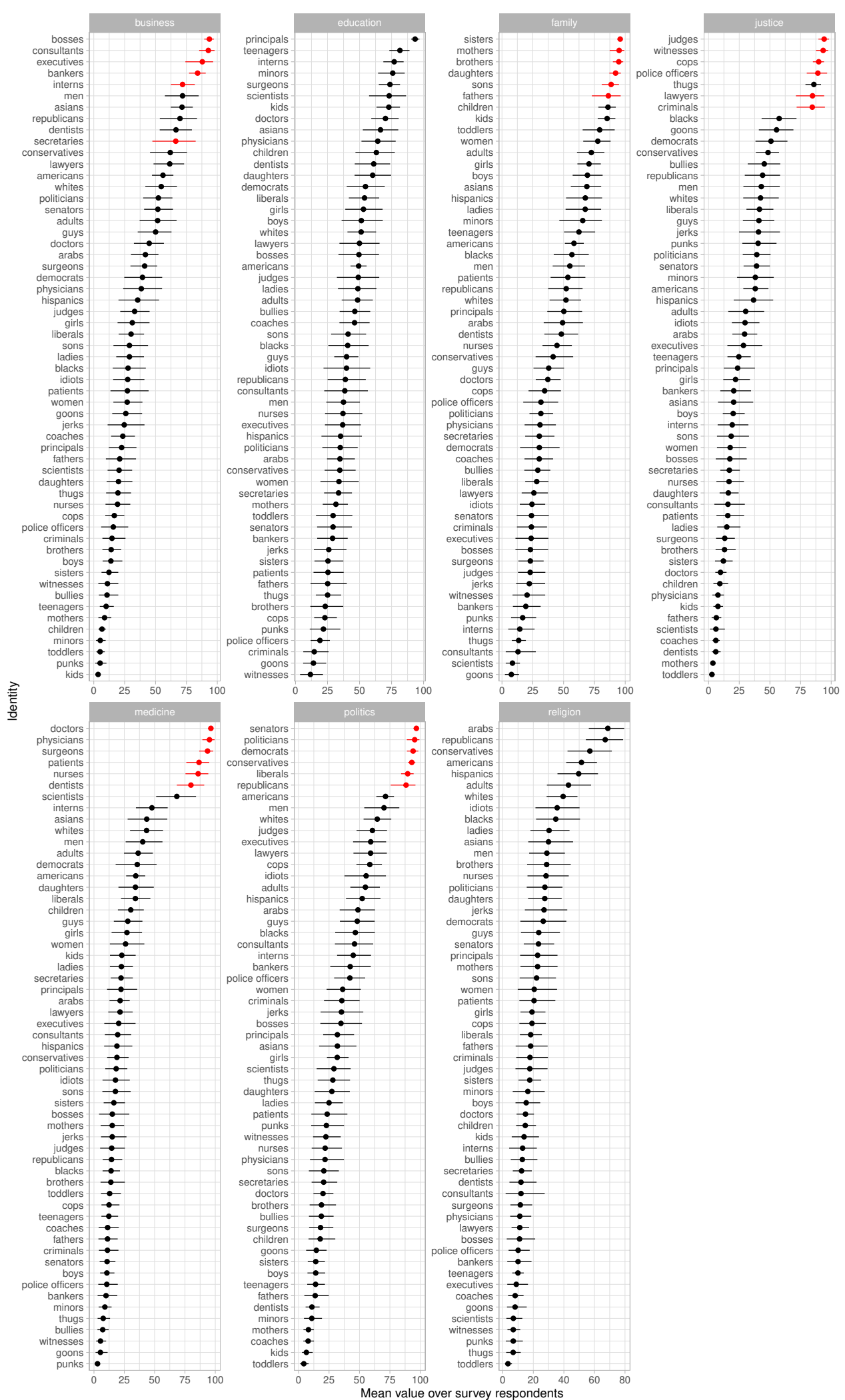

Figure 7: Associations for each identity to each of the institutional settings we study. Results are given with $95 \%$ bootstrapped confidence intervals, and each institutional setting is a different subplot. Red estimates represent identities that were assumed to be clustered on the given institutional setting 

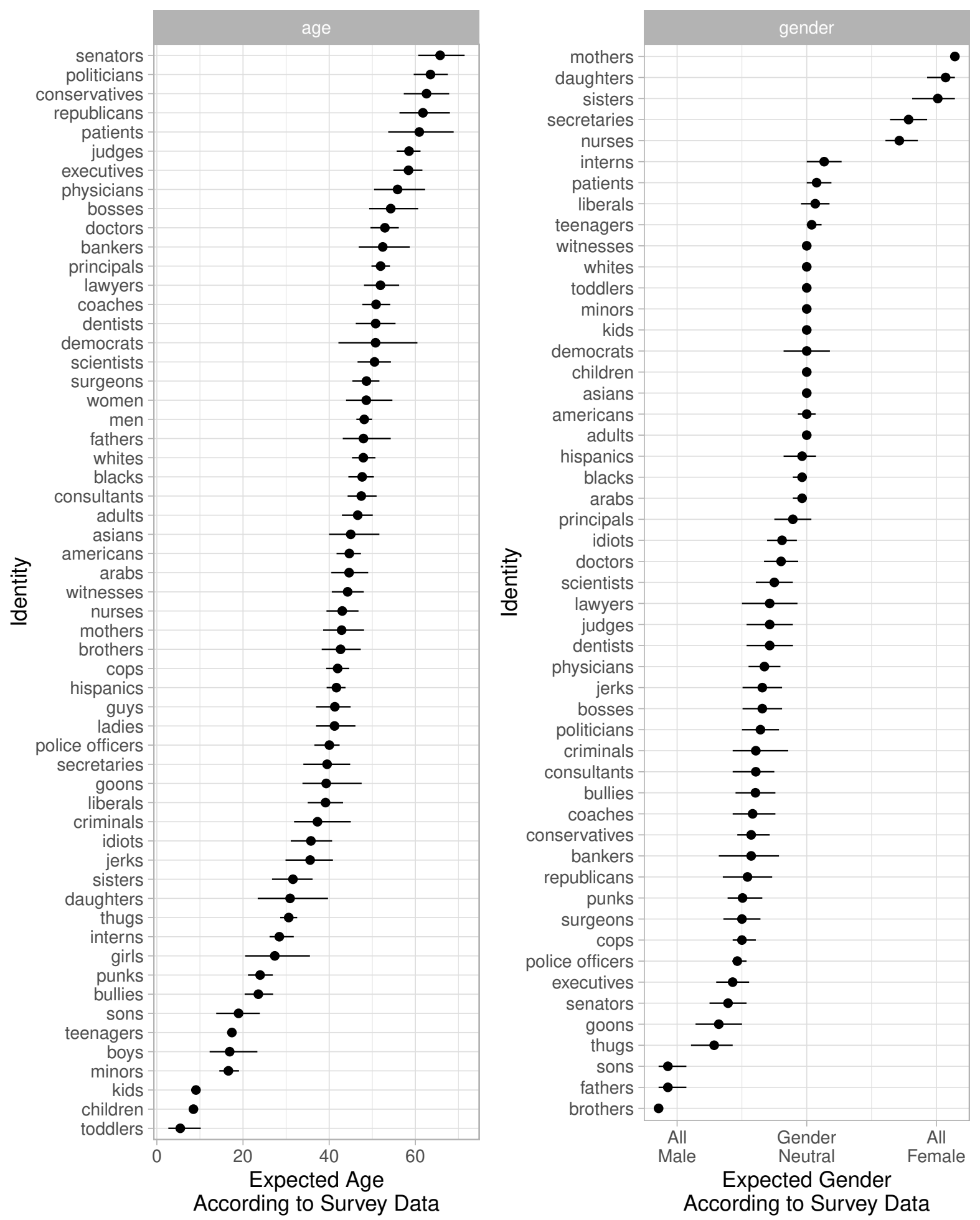

Figure 8: On the left, 95\% confidence intervals for the expected age of each identity. On the right, $95 \%$ confidence intervals for the expected gender of each identity, assuming a continuous dimension for gender. Note the gender labels are slightly shifted inward so labels are readable, meaning some points extend beyond the label itself. 

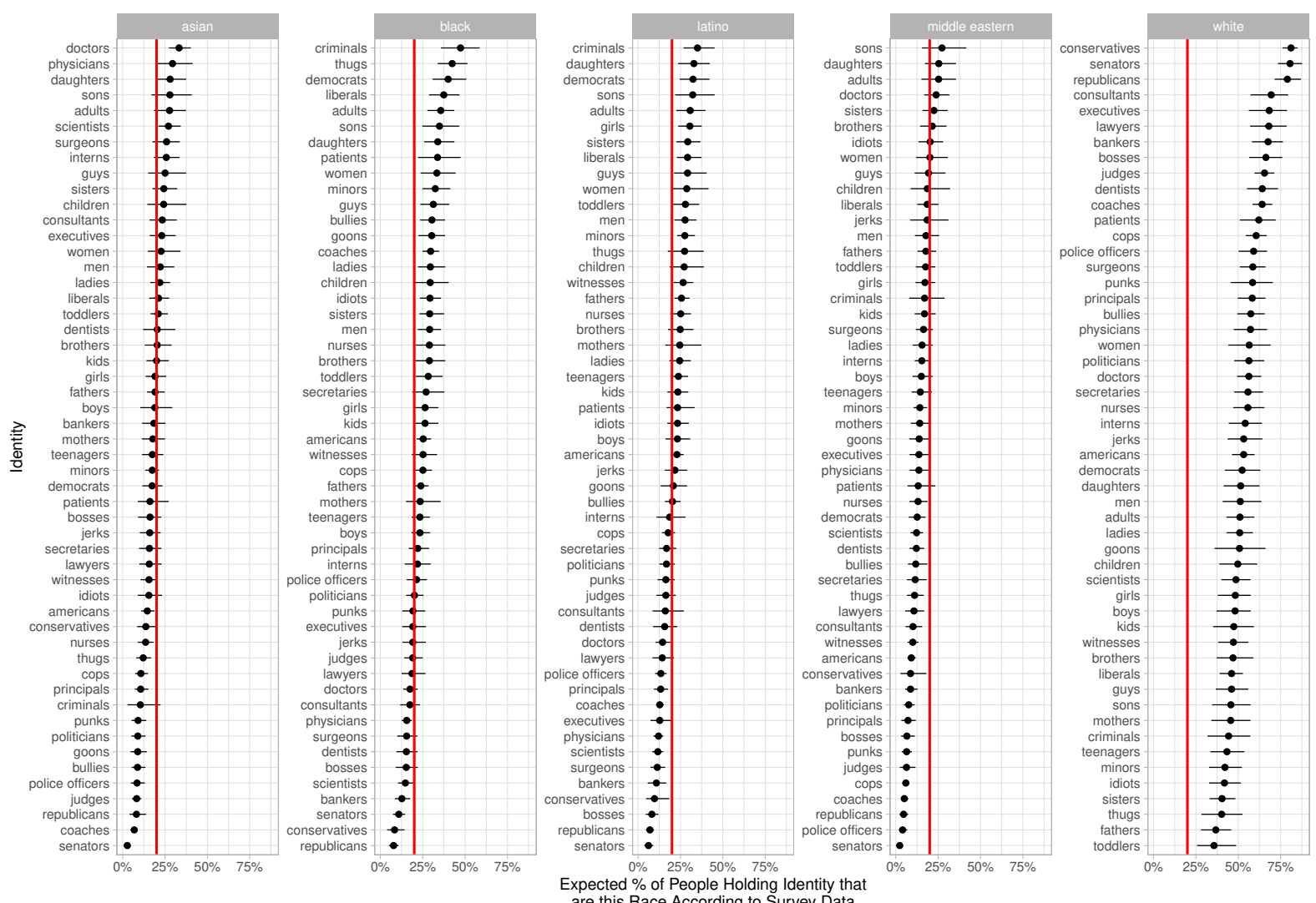

Figure 9: 95\% confidence intervales for the perceived percentage of each identity that are also of a given race. The five racial/ethnic categories we consider are represented as separate subplots, the red line at $20 \%$ gives the expected value of all races were equally represented for the identity. 


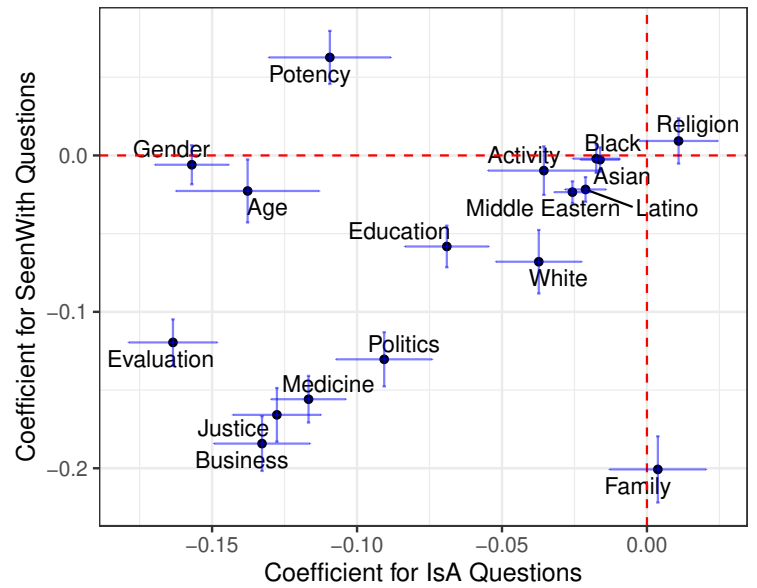

Figure 10: Coefficients for the regression models described in the main text for IsA and SeenWith questions for each dimension. Blue bars represent error bars for each dimension on IsA questions (horizontal) and SeenWith (vertical). Red dotted lines represent zero, any error bars that cross this line are not significant 

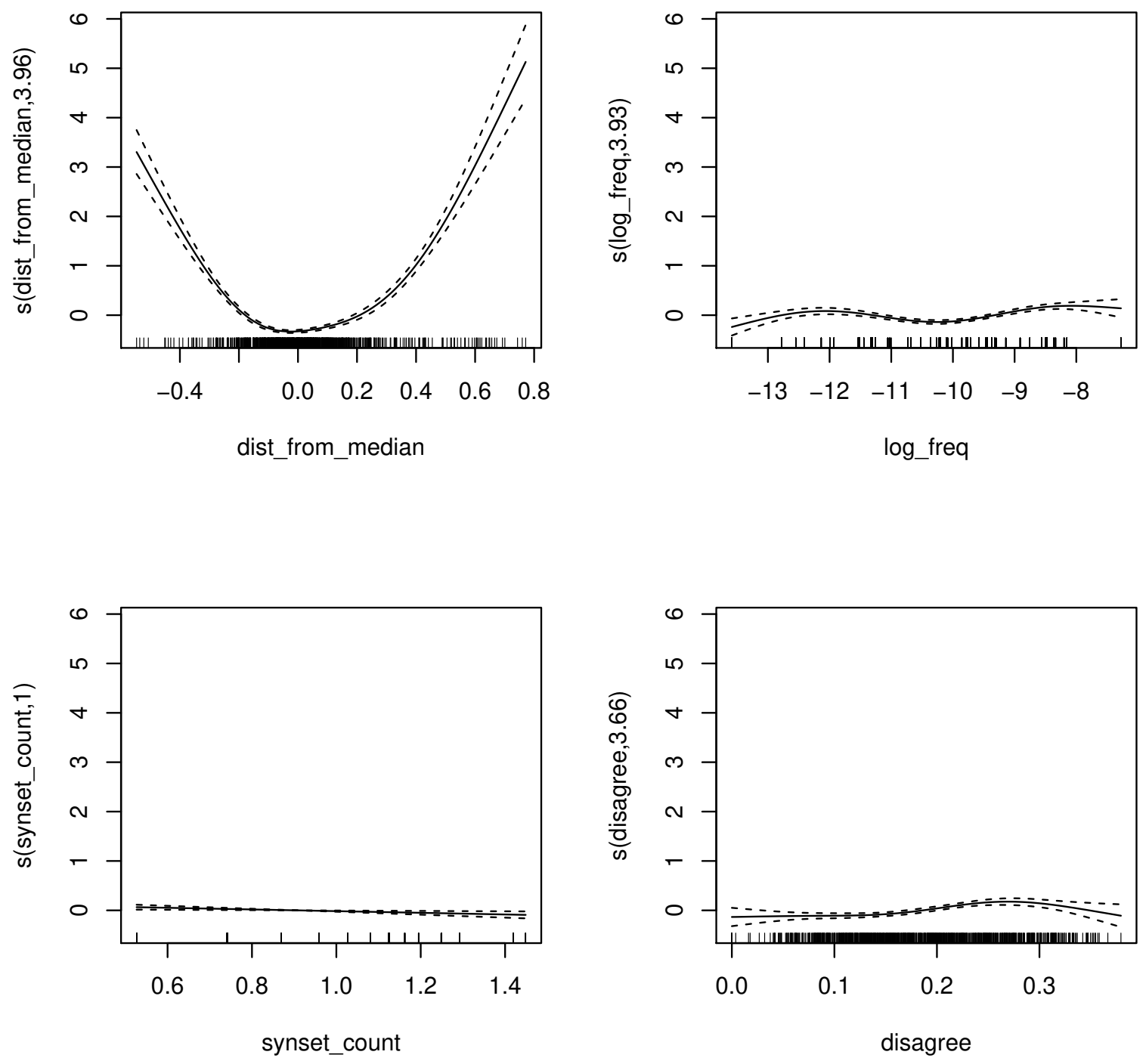

Figure 11: Regression results for survey data from this paper on the belief-level measure 

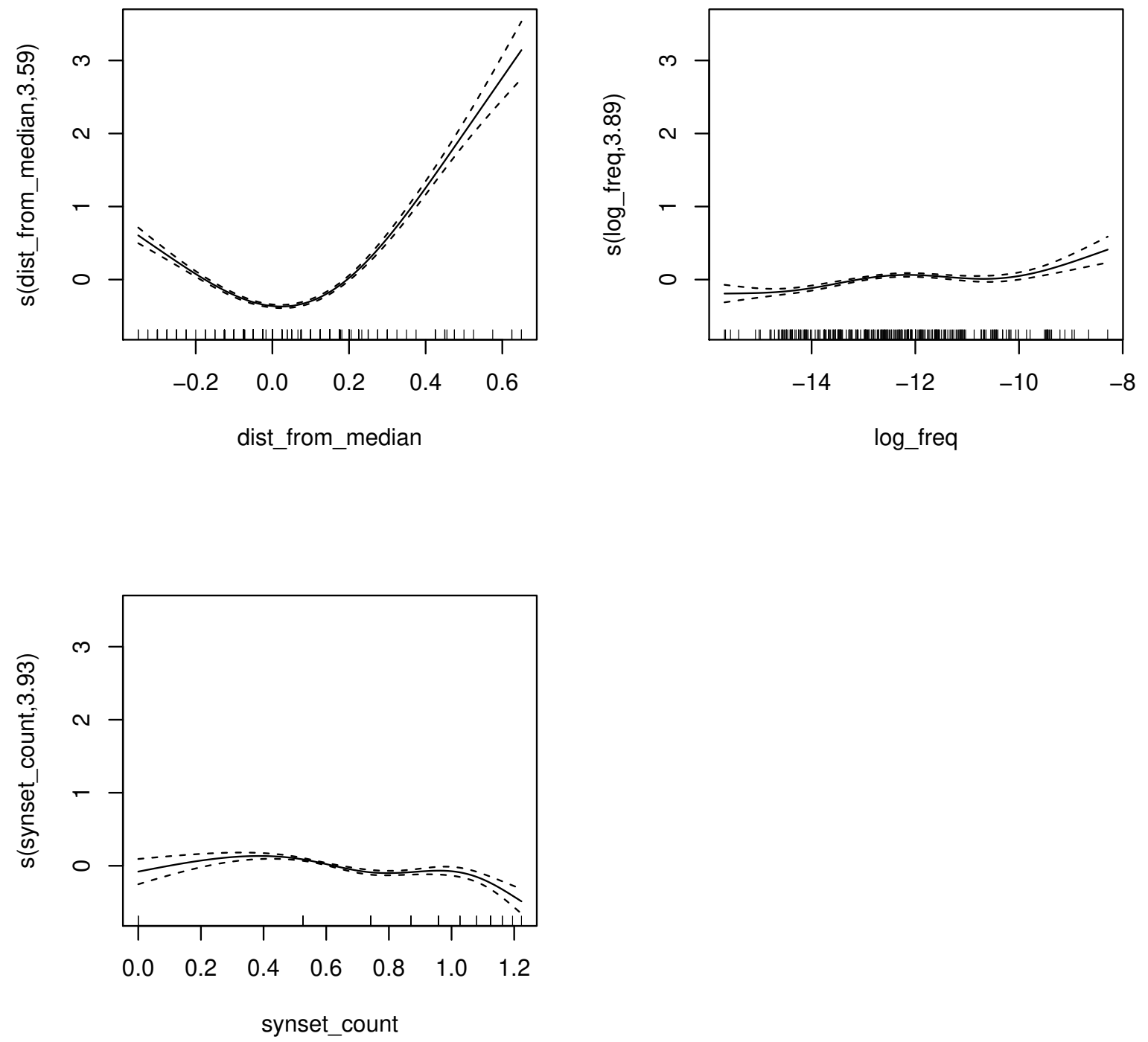

Figure 12: Regression results for survey data from Bolukbasi et al. (2016) on the belief-level measure 

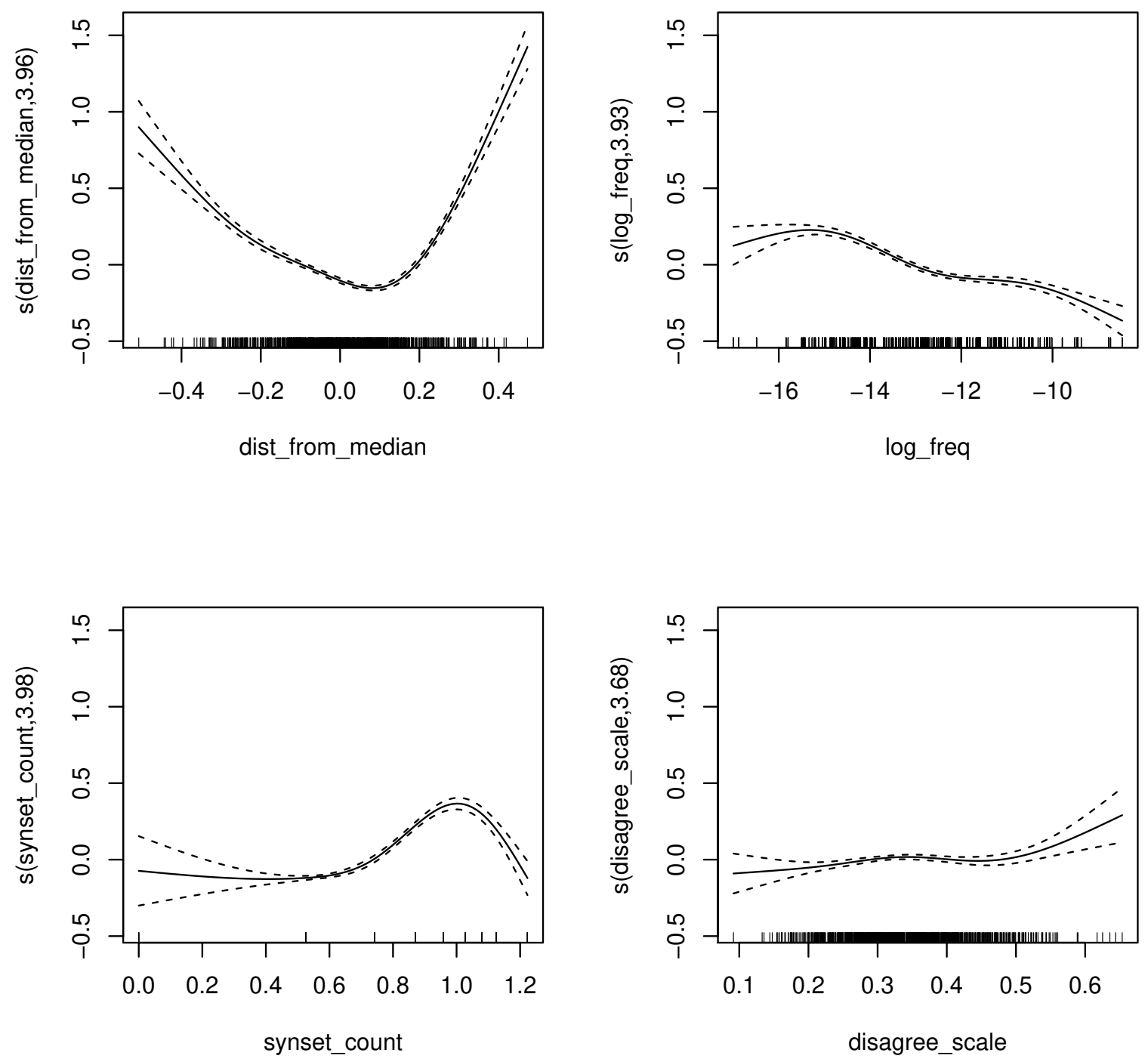

Figure 13: Regression results for survey data from Agarwal et al. (2019) on the belief-level measure 

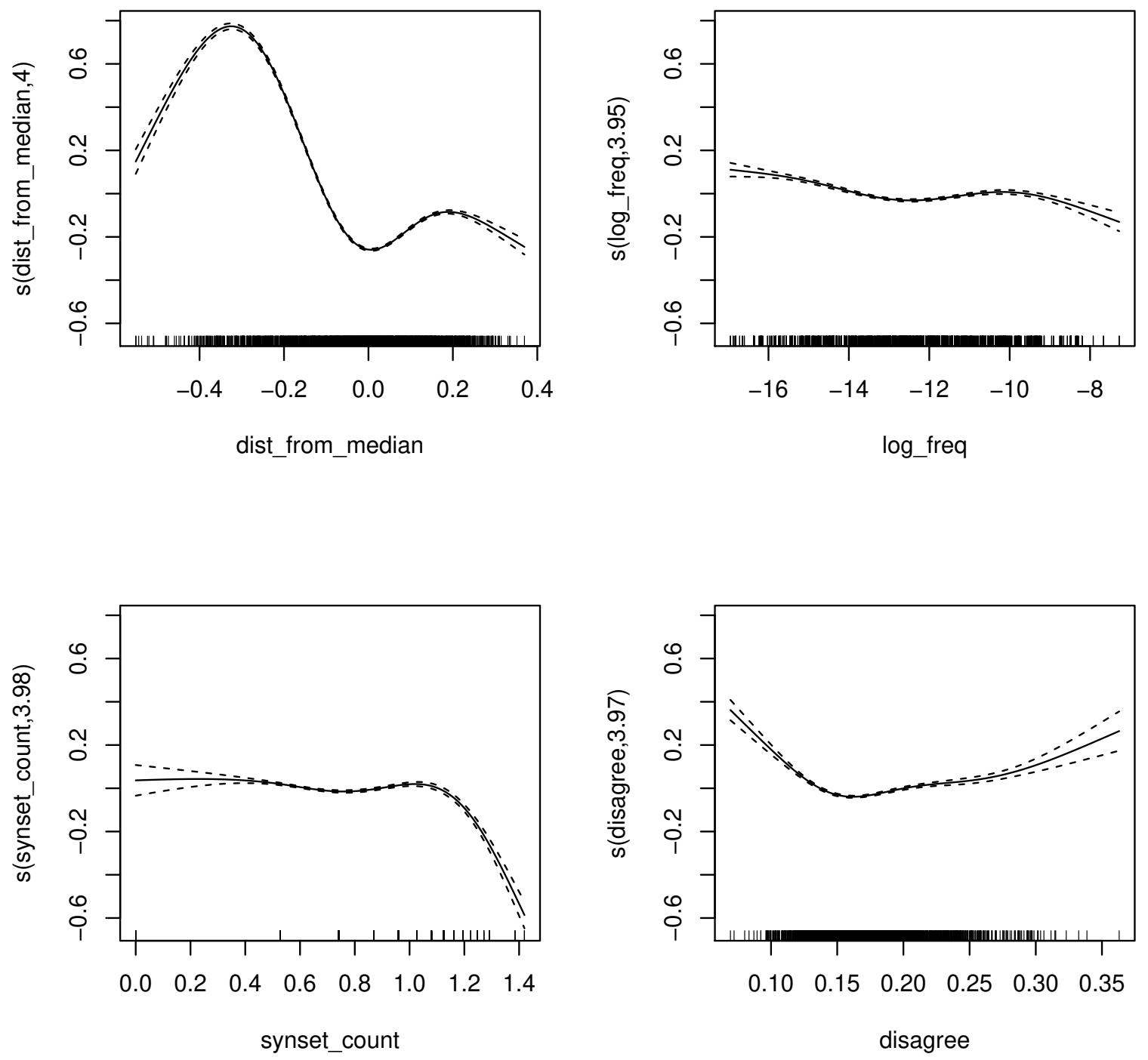

Figure 14: Regression results for survey data from Smith-Lovin and Robinson (2015) on the belief-level measure 